\title{
The Influence of Odevity of Carrier Ratio on Three-level Rectifier Wang Pengzhan ${ }^{1}$, a Luo Wei $^{2}$, Yang Shasha ${ }^{1}$, Cao Tianzhi ${ }^{3}$ and Li Huawei ${ }^{1}$ \\ ${ }^{1}$ School of Electrical Engineering, Beijing Jiaotong University, Beijing 100044, China. \\ 2 State Grid Jibei Electric Power Company Limited, Beijing 100045,China. \\ ${ }^{3}$ Electric Power Research Institute,State Grid Jibei Electric Power Company Limited, Beijing100045, China. \\ a14121469@bjtu.edu.cn
}

Keywords: Three level rectifier, carrier ratio, transient current control, neutral point voltage, harmonic analysis.

\begin{abstract}
Neutral point voltage balancing is an inherent problem of three-level rectifier. This paper first analyzed the cause to the neutral point voltage deviation of the DC-side three-level rectifier, and studied the charge and discharge transient processes of the two DC-side capacitors and the harmonic characteristics of the current of the AC-side rectifier respectively when the carrier ratio is an odd number or even number. It also verified its studies through simulation. The results showed that, when the carrier ratio is an even number, the neutral point voltage of the three-level rectifier is more easily to be balanced and will not cause bad influence on the harmonic characteristics of the AC-side rectifier.
\end{abstract}

\section{Introduction}

Single-phase three-level rectifier should assure the voltage balance of the two series DC-side capacitors. There are mainly two factors that may cause unbalanced neutral point voltage: incomplete consistency of the capacitors of the two supporting DC-side capacitors and asymmetric charge and discharge transient processes on the two capacitors. There are normally two solutions. The first method is to connect the two ends of the capacitor to an isolating DC power ${ }^{[1-2]}$; the second method is to change the conduction time of the power device to make the average current zero when passing the neutral point ${ }^{[3-4]}$. Both of the two solutions require extra devices or control strategies to keep a balanced voltage of the two capacitors.

Single-phase three-level pulse rectifier is usually controlled by carrier phase-shifting SPWM ${ }^{[5]}$. Literature [6] stated that too low carrier frequency may cause unbalanced voltage of the capacitor, but has no differentiation on the selection of carrier ratio; Literature [7] stated that the harmonic elimination between the two phases of three-phase inverter has no relation with carrier ratio, but did not extend to single-phase three-level inverter. Based on the analysis on the charge and discharge processes of the two DC-side capacitors of the single-phase three-level pulse rectifier, the paper found that the charge and discharge processes of the two capacitors are related with the odevity of the carrier ratio; then the paper analyzed the influences of different selections of odevity of the carrier ratio on the neutral point voltage balancing on the $\mathrm{DC}$ side and the current harmonics on the AC side, and verified the theoretical analysis through simulation.

\section{Topological structure and working model of single-phase three-level rectifier}

The topological structure of the main circuit of single-phase three-level rectifier is shown as Fig. 1. $\mathrm{u}_{\mathrm{N}}$ represents the output voltage of the transformer secondary side traction winding; $\mathrm{U}_{1}$ and $\mathrm{U}_{2}$ represent the voltages of DC-side capacitor $\mathrm{C}_{1}$ and capacitor $\mathrm{C}_{2}$. The two rectifier bridge arms consist of eight IGBT elements. If the switch elements of each bridge arm are replaced by an ideal three-level switch, the switch equivalent circuit can be obtained as shown in Fig. 2. 


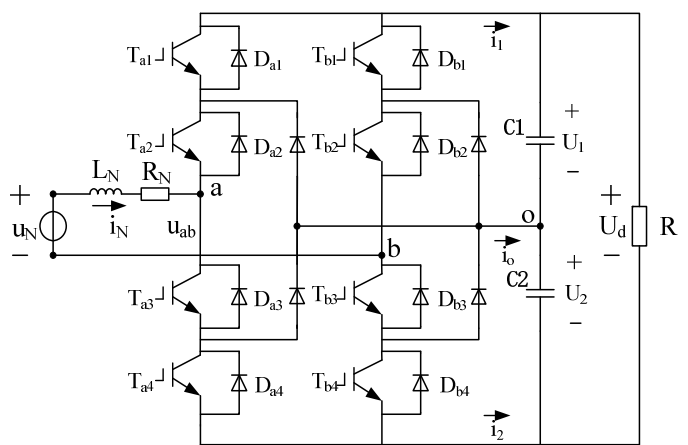

Fig. 1 Topological structure of single- phase three-level rectifier

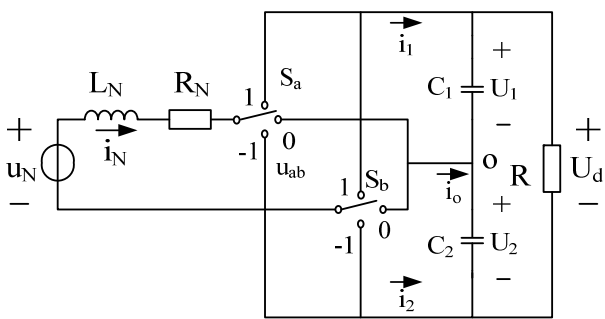

Fig.2 Switch equivalent circuit of single-phase three-level rectifier

$S_{\mathrm{a}}$ and $\mathrm{S}_{\mathrm{b}}$ are defined as the ideal switch functions, which represent the switch status of two bridge arms. Each switch function includes three values $(-1,0,1)$. According to different controlling strategies, the rectifier can change its working mode under certain rules.

\section{The influence of odevity of carrier ratio on neutral point voltage balancing}

\subsection{Causes to neutral point voltage deviation of single-phase three-level rectifier}

when any of the bridge arm switches is a zero level, deviation of the neutral point voltage on the DC side will occur and lead to unbalanced voltages of the two capacitors. The voltage deviation of the two capacitors can be expressed as:

$$
\Delta \mathrm{U}=\mathrm{U}_{1}-\mathrm{U}_{2}=-\frac{1}{\mathrm{C}} \int \mathrm{i}_{0} \mathrm{dt}+\mathrm{k}
$$

Where $K$ stands for the integration constant, and the neutral point current $i_{0}=-\left(S_{a}^{2}-S_{b}^{2}\right) i_{N}$. When $\mathrm{S}_{\mathrm{a}}^{2}-\mathrm{S}_{\mathrm{b}}^{2} \neq 0$, a deviation between the voltages of the two capacitors will occur. When adopted with unipolar PWM modulation for the $\mathrm{k}$ switching period, the peak pulse voltage on the DC side will be:

$$
\Delta \mathrm{u}_{\mathrm{k}}=\left|\mathrm{I}_{\mathrm{m}} \sin \left(\omega \mathrm{kT} \mathrm{T}_{\mathrm{s}}\right)\right|\left[\mathrm{t}_{\mathrm{on}}\left(\mathrm{T}_{\mathrm{s}}-\mathrm{t}_{\mathrm{on}}\right) / \mathrm{CT}_{\mathrm{s}}\right]
$$

Where, $\mathrm{T}_{\mathrm{s}}$ is the switch period, $\mathrm{I}_{\mathrm{m}} \sin \left(\omega \mathrm{kT}_{\mathrm{s}}\right)$ is the line side current value of the $\mathrm{k}$ switch period, and $t_{o n}$ is the switch conduction time. There are mainly four types of working modes:

Table 1 four types of working modes of neutral point voltage deviation

\begin{tabular}{|r|r|c|c|c|c|c|}
\hline $\mathrm{S}_{\mathrm{a}}$ & $\mathrm{S}_{\mathrm{b}}$ & $\mathrm{u}_{\mathrm{ab}}$ & \multicolumn{2}{|c|}{$\mathrm{u}_{\mathrm{N}}>0$} & \multicolumn{2}{c|}{$\mathrm{u}_{\mathrm{N}}<0$} \\
\hline & & & Energy transfer & Status & Energy transfer & Status \\
\hline 1 & 0 & $\mathrm{U}_{\mathrm{d}} / 2$ & $\mathrm{u}_{\mathrm{N}} \rightarrow \mathrm{C}_{1}$ & Charging & $\mathrm{C}_{1} \rightarrow \mathrm{u}_{\mathrm{N}}$ & Discharging \\
\hline 0 & 1 & $-\mathrm{U}_{\mathrm{d}} / 2$ & $\mathrm{C}_{1} \rightarrow \mathrm{u}_{\mathrm{N}}$ & Discharging & $\mathrm{u}_{\mathrm{N}} \rightarrow \mathrm{C}_{1}$ & Charging \\
\hline 0 & -1 & $\mathrm{U}_{\mathrm{d}} / 2$ & $\mathrm{u}_{\mathrm{N}} \rightarrow \mathrm{C}_{2}$ & Charging & $\mathrm{C}_{2} \rightarrow \mathrm{u}_{\mathrm{N}}$ & Discharging \\
\hline-1 & 0 & $-\mathrm{U}_{\mathrm{d}} / 2$ & $\mathrm{C}_{2} \rightarrow \mathrm{u}_{\mathrm{N}}$ & Discharging & $\mathrm{u}_{\mathrm{N}} \rightarrow \mathrm{C}_{2}$ & Charging \\
\hline
\end{tabular}

The voltage pulse value of the $\mathrm{k}$ switch period on the $\mathrm{DC}$ side is determined by the line side current value and the conduction time of the period.

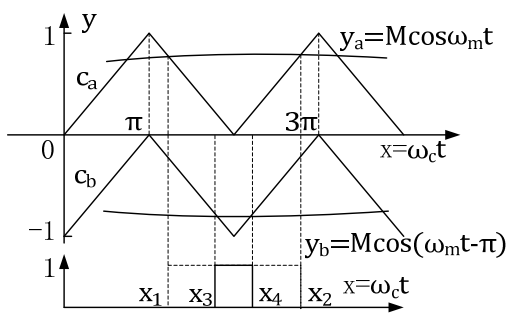

Fig.3 Unit cell for double-edge triangular carrier modulation

In the above figure, $\mathrm{M}$ stands for the modulation depth; $\mathrm{c}_{\mathrm{a}}$ and $\mathrm{y}_{\mathrm{a}}$ stand for the carrier wave and modulation wave at the positive side of the bridge arm $a ; c_{b}$ and $y_{b}$ stand for the bridge arm $b$. In a carrier wave period, the modulation wave is usually set to a fixed value. In a modulation wave period, the regions that satisfy switch combinations of $S_{a}=1$ and $S_{b}=0$ are the parts from $x_{3}$ to $x_{4}$, the pulse width is $x_{4}-x_{3}=2 \pi-2 \pi M \cos \omega_{m} t$. When $S_{a}=0, S_{b}=-1$, the pulse widths are $2 \pi-2 \pi M \cos \omega_{m} t$, $\omega_{0} t \in[0, \pi]$. Based on the symmetrical modulation methods, the pulse widths under two switch function combinations of $(0,1)$ and $(-1,0)$ are $2 \pi+2 \pi M \cos \omega_{m} t, \omega_{m} t \in[\pi, 2 \pi]$. Hence, the pulse 
width under four switch combinations can be expressed by $2 \pi+2 \pi M\left|\cos \omega_{m} t\right|$ with the change period of $\pi$. Therefore, in a modulation wave period, the value of $\Delta \mathrm{u}_{\mathrm{k}}$ changes with the period of $\pi$.

3.2 The charge and discharge status of the two DC-side capacitors when the carrier ratio is an even number

In the simulation model, the DC-side voltage and AC-side voltage are $1500 \mathrm{~V}$ and $3000 \mathrm{~V}$ respectively; the two DC-side capacitors have the same capacity.

Assume the voltage change of the DC-side capacitor $\mathrm{C}_{1}$ is $\Delta \mathrm{u}_{\mathrm{kC} 1}$, and the voltage change of the capacitor $\mathrm{C}_{2}$ is $\Delta \mathrm{u}_{\mathrm{kC} 2}$. In a modulation period $\mathrm{T}$, when the carrier ratio is an even number, the working model that is half a period away from the working mode of $(1,0) /(0,-1)$ will be $(-1,0)$

$/(0,1)$. That is to say, no matter in the status of charging or discharging, for any voltage change $\Delta \mathrm{u}_{\mathrm{kC} 1}$ of the capacitor $\mathrm{C}_{1}$ in the positive half period, there is a corresponding $\Delta \mathrm{u}_{\mathrm{kC} 2}$ in the negative half period, with a distance of $\mathrm{T} / 2$; similarly, any $\Delta \mathrm{u}_{\mathrm{kC} 2}$ in the positive half period has an equivalent $\Delta \mathrm{u}_{\mathrm{kC} 1}$ in the negative half period.

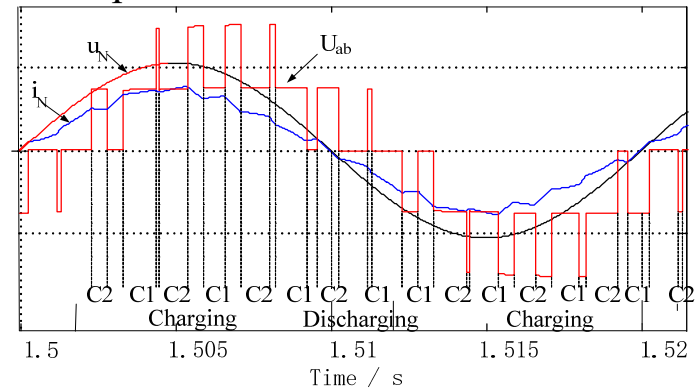

Fig. 4 The charge and discharge status of the two DC-side capacitors (carrier ratio $\mathrm{N}=8$ )

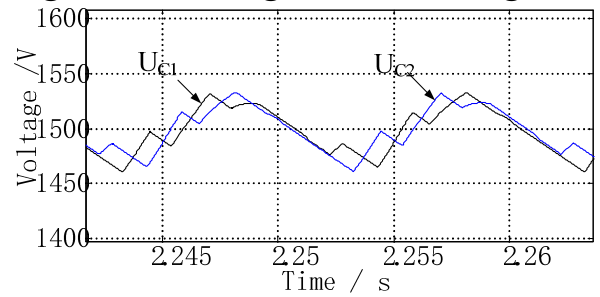

Fig. 5 The voltage waveforms of the two DC-side capacitors (carrier ratio $\mathrm{N}=8$ )

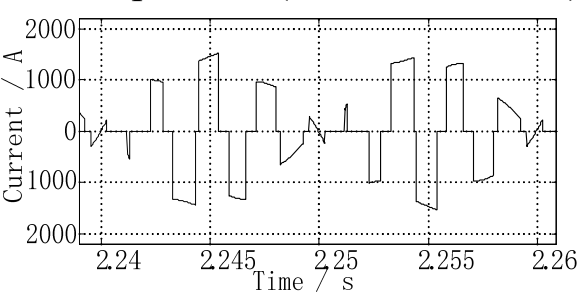

Fig.6 The neutral point current waveform on the DC side (carrier ratio $\mathrm{N}=8$ )

As the voltage pulse frequency on the DC side is twice of the fundamental frequency, two DC voltage ripples will be produced in one modulation wave period. The voltage change curve of the modulation wave in the positive half period $\mathrm{C}_{1}$ is the same as that of the negative half period $\mathrm{C}_{2}$, and the voltage change curve of the modulation wave in the positive half period $\mathrm{C}_{2}$ is the same as that of the negative half period $\mathrm{C}_{1}$. Hence, in a modulation wave period, the capacitor and $\mathrm{DC}$-side capacitor have equivalent quantity of charge without unbalanced voltage.

According to the neutral point current, the waveform of the neutral point current in the later latter half period is the upside down of the first half period. The total current impulse of a modulation wave period is 0 , that is $\int \mathrm{i}_{0} \mathrm{dt}=0$.

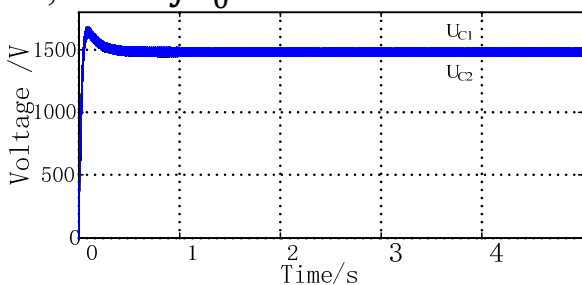

Fig. 7 The voltage waveforms of the two

DC-side capacitors (carrier ratio $\mathrm{N}=8$ )

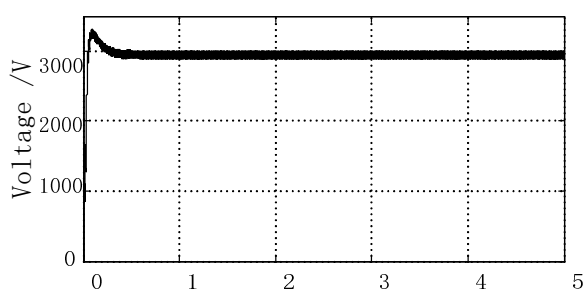

Fig. 8 The voltage waveform on the DC side (carrier ratio $\mathrm{N}=8$ )

When the carrier ratio is an even number, the voltages of the two DC-side capacitors are basically equivalent, which can help the neutral voltage keep balanced. 


\subsection{The charge and discharge status of the two DC-side capacitors when the carrier ratio is an odd number}

When the carrier ratio is an odd number, in a modulation wave period, the mode at a distance of $\mathrm{T} / 2$ from the operating mode $(1,0) /(0,-1)$ is the $(0,1) /(-1,0)$ mode. In other words, there are no one-to-one correspondences between the voltage variable $\Delta \mathrm{u}_{\mathrm{kC} 1}$ of capacitor $\mathrm{C}_{1}$ and the voltage variable $\Delta \mathrm{u}_{\mathrm{kC} 2}$ of capacitor $\mathrm{C}_{2}$ within a modulation wave period.

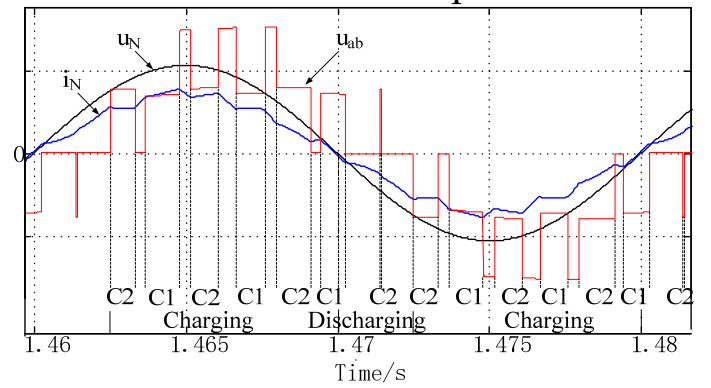

Fig. 9 The charge and discharge status of the DC-side current (carrier ratio $\mathrm{N}=9$ )

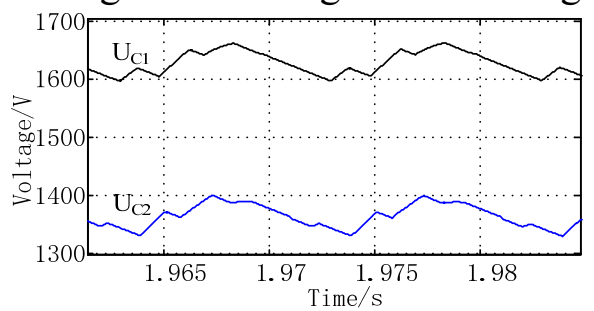

Fig. 10 The charge/discharge status of the $\mathrm{DC}$-side current (carrier ratio $\mathrm{N}=9$ )

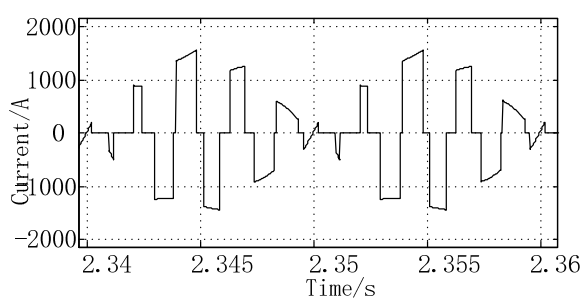

Fig. 11 The waveform of DC-side midpoint current (carrier ratio $\mathrm{N}=9$ )

As shown in the figures, the voltage ripples of $\mathrm{C}_{1}$ and $\mathrm{C}_{2}$ are inconsistent. The obtained charges on $\mathrm{C}_{1}$ and $\mathrm{C}_{2}$ within a modulation wave period may have some deviations due to the undefined location of $\varphi$,these deviations would increase gradually with time and eventually become irreversible. In this case, the voltages of the two DC-side capacitors could be seriously unbalanced.

The neutral point current presents no distinct symmetric relation in both the plus and minus intervals, it cannot be ensured that the impulse within a modulation wave period is 0 .

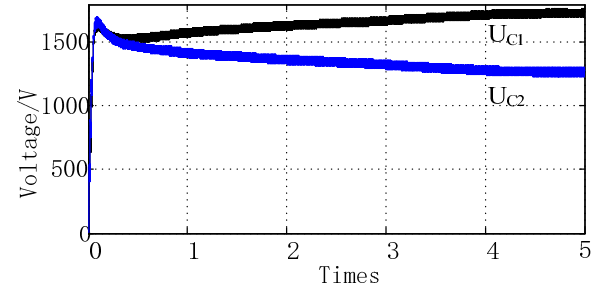

Fig. 12 The waveform of two DC-side capacitors' voltage (carrier ratio $\mathrm{N}=9$ )

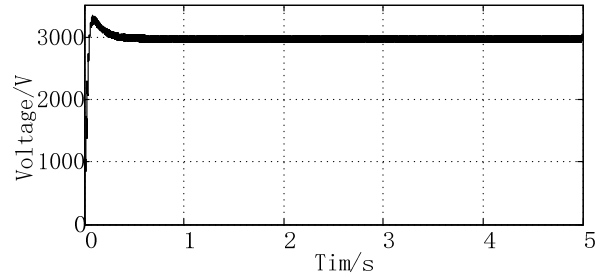

Fig. 13 The waveform of DC-side voltage (carrier ratio $\mathrm{N}=9$ )

When the carrier ratio is an odd number, the charges obtained by the two supporting capacitors within a modulation wave period are different. Although the general DC-side voltage can remain steady within a certain time period, the neutral point voltage will be deviated due to unbalanced voltage distribution of the two capacitors.

\section{Influence of the odevity of the carrier ratio on the AC-side current of the rectifier}

The distortion degree of the output current waveform to the ideal sinusoidal waveform is an important measurement index of converter's modulation effects. In this research, the current harmonics on the AC side are selected as the study object. 


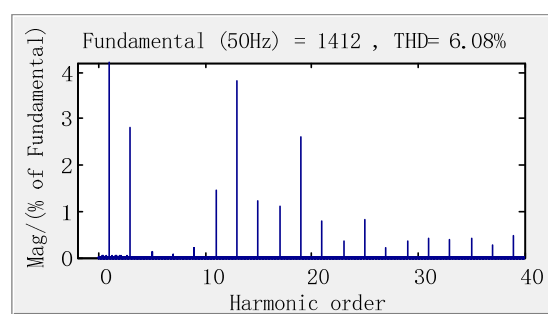

Fig. 14 The harmonic content of the grid-side current (carrier ratio $\mathrm{N}=8$ )

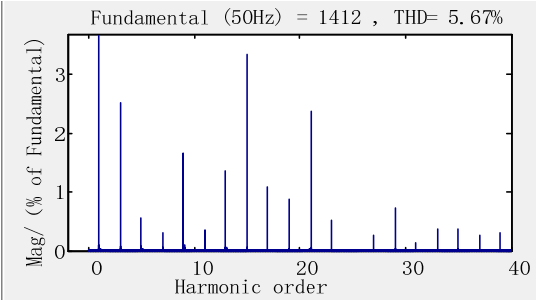

Fig. 15 The harmonic content of the grid-side current (carrier ratio $\mathrm{N}=9$ )

When the carrier ratio is 8 , the numbers of harmonic waves are $13,15,17$, and 19 . When the carrier ratio is 9 , the numbers of harmonic waves are $15,17,19$, and 21 . The harmonic waves are mainly concentrated on the odd-numbered sidebands close to the even switching frequencies and even-numbered harmonic waves does not exist no matter the carrier ratio $\mathrm{N}$ is an odd number or even number. Furthermore, the amplitude of each harmonic wave is only related to the value of $U_{d}, \mathrm{M}, \mathrm{m}$, $\mathrm{n}$, and the carrier ratio; it has no direct relationship with the odevity of carrier ratio.

\section{Conclusions}

In this research, by study the charge and discharge process of the DC-side capacitors on the consumption that the two DC-side supporting capacitors are equivalent. The findings reveal that the odevity of the chosen carrier ratio has influence on the charge and discharge transient process of the two capacitors. And the theoretical analysis suggests that if the carrier ratio is an odd number, it cannot ensure the consistency of the two capacitors' charge and discharge process; it may lead to the excursion of neutral point voltage. If the carrier ratio is an even number, the charge and discharge process of the two DC-side capacitors will present consistency; in this case, the impulse of the total current within a modulation wave period will be 0 . According to the analysis of the current's harmonic property on the AC side, the odevity of carrier ratio has no influence on harmonic property; the selection of even-numbered carrier ratio will not lead to extra even-numbered harmonic wave. Additionally, all the above findings are proved to be correct with simulation experiments, which have certain guiding influence on the selection of rectifier carrier frequency.

\section{References}

[1] Yazdani A, Iravani R A. Generalized state-space averaged model of the three-level NPC converter for systematic DC-voltage-balancer and current-controller design[J]. IEEE Transactions on Power Delivery, 2005, 20(2)1105-1114.

[2] Menzies R W, Steimer P, Steinke J K. Five level GTO inverter for large induction motor drives[J]. IEEE Transactions on Industry Applications, 1994, 30(4) 938-944.

[3] Jiang Weidong, Yang Bowang, Huang Jing, et al. Comparisons of the Neutral Point Voltage Balancing Algorithm for NPC Three-level Inverters Based on Different Zero-sequence Voltage Injection[J].Proceedings of the CSEE, 2013, 33(3) 17-25.

[4] Wang Jianyuan, Lei Guohui, Zhong Yanru. Research on one hysteresis control + precise control strategy of three-level inverter for neutral - point potential balance[J]. Electric Machines and Control, 2015, 19(7) 66-72.

[5] Gray W.Chang, Hsin-Wei Lin, Shin-Kuan Chen. Modeling Characteristics of Harmonic Currents Generated by High-Speed Railway Traction Drive Converters[J].IEEE Transactions on Power Delivery, 2004, 19(2) 766-773.

[6] Yuan Zhichang, Song Qiang, Liu Wenhual. Selection of phase-shift SPWM switching frequency[J]. Electric Power Automation Equipment, 2009, 29(8) 37-41. 
[7] D.Grahame Holmes. Pulse Width Modulation for Power Converters [M]. 2003: 251-253. 